\title{
BIONETS: BIO-inspired NExt generaTion networkS
}

\author{
Iacopo Carreras, Imrich Chlamtac, Hagen Woesner, and Csaba Kiraly \\ CREATE-NET, Trento, Italy \\ \{carreras, woesner, chlamtac, kiraly\}@create-net.it \\ http://www.create-net.it
}

\begin{abstract}
The amount of information in the new emerging all-embracing pervasive environments will be enormous. Current Internet protocol conceived almost forty years ago, were never planned for these emerging pervasive environments. The communications requirements placed by these protocols on the low cost sensor and tag nodes are in direct contradiction to the fundamental goals if these nodes, being small, inexpensive and maintenance free. This situation needs therefore a radically different approach to communication in these systems, especially since pervasive and ubiquitous networks are expected to be the key drivers of the all encompassing Internet of the coming decades. The fundamental disparity between the need for extremely dispensable, low cost devices, such as sensors or tags, and increasing communications load per device due to the presence of billions of nodes, that is creating an unbridgeable paradox, is therefore an insurmountable obstacle on the way to adoption when conventional networking architectures are being considered. Biological systems provide insights into principles which can be adopted to completely redefine the basic concepts of control, structure, interaction and function of the emerging pervasive environments. The study of the rules of genetics and evolution combined with mobility, leads to the definition of service oriented communication systems which are autonomous, and autonomously self-adaptive. The objective of this article is to ascertain how this paradigm shift, which views a network only as a randomly self-organizing by-product of a collection of self-optimizing services, may become the enabler of the new world of omnipresent low cost pervasive environments of the future.
\end{abstract}

\section{Introduction}

As the trend toward ubiquitous and pervasive computing continues to gain momentum, the number of nodes is expected to grow by multiple orders of magnitude as tags, sensors, body networks and myriad of other miniaturized devices get fully integrated into the global communication superstructure. Not only will the amount of information in these all-embracing pervasive environments be enormous and to a large degree localized, but also the ambiance within which these nodes will act will be intelligent, mobile, self- cognitive, knowledge based, 
and, in a sense, "almost alive". Current Internet protocols, conceived almost forty years ago, were never planned for these emerging pervasive environments. Besides their many limitations with respect to agility, mobility, scalability, etc., they are also too heavyweight in the computing and communication requirements they impose on the small and energy- limited nodes. Hence a totally new way of controlling these emerging systems is needed. Realizing that the raison d'etre for the protocol layers is the support of better services to the user, we consider an approach to the pervasive environments that is derived from the network's original goal - services. Given, as observed above, that the pervasive systems are dynamic, growing, self aware and evolving, it is natural to model them as biological entities. Considering the behavior and hence control of these pervasive environments an evolving organism leads us to applying the rules of combination of genetic material and evolution to determine the information exchange, fully replacing the concept of end to end Internet oriented protocols. By information exchange occurring in these systems only as an individual need of each entity in the biosystem to reproduce, the rules of control become localized, minimized and occur only when and where needed, making it possible for the individual nodes to work with minimal energy, with computing or communication effort expended only as it benefits their own goals. Even more important, by defining themselves as the end result of the evolution process, the services here become characterized by their ability to mutate and select the fittest to survive, this way constantly evolving and self-optimizing leading to a new concept of self aware, self optimizing bionetwork, a candidate for the new world of omnipresent low cost pervasive environments of the future. This article expands on our previous work published in [1 in defining the processes and mapping of the biological approach to pervasive systems communication.

\section{Autonomic Pervasive Environment Today}

In the new emerging pervasive environments the rules of the game are drastically changing. In previous systems, mobility was something that needed to be handled in order to extend the already existing services to a wireless context. Nowadays, almost every person has a cell phone and almost every person is using the wireless devices for more and more purposes which are not just phone calls. Services are accessed from remote locations, while moving and without a reliable end-to-end connection. In such a new moving environment, it is natural to start asking which would be the best way to benefit from this implicit user mobility and how to maximally exploit it.

In the traditional Internet the flow of information follows the source-todestination philosophy. Within the last years two main research directions evolved that cover the world of pervasive networking: Mobile ad-hoc networks (MANETs) and Wireless Sensor Networks (WSN). While the MANETs try to cope with mobility, the research in WSN concentrated on dealing with huge numbers of 
power-constraint data sources. Using sensor nodes for relaying packet data from other sensors turned out to be a serious power drain, limiting the life time of sensors. In [2] 3] the authors introduce hierarchy into previously flat sensor network architectures. Here, the users carry data packets collected from sensors or other users and drop it whenever they encounter a gateway to the backbone. This way mobility can be exploited to provide connectivity in a disconnected environment. The information is treated exactly as in the flat networks, but with a different routing policy. Services are the same as well as the entire protocol stack. The same idea of using mobility to create connectivity was shown for MANETs in 4. In 5 the authors show that mobility indeed increases the capacity of MANETs.

All the previously described networks deal with the transport of data packets, and while there are techniques to do data fusion in WSN, their aim is to reduce the number of data packets to forward by eliminating redundancy.

\subsection{Network Original Goal Is Service}

Trying to find a better solution to the exposed problem this article totally changes the perspective. In the new ubiquitous context the information that is managed and exchanged by users is drastically changing in its significance. Information will not only be to a high degree localized, but also aging, which means that most of the time information will simply be outdated and therefore useless with respect to the context where the user is moving in. In this sense, source-to-destination data transport will be needed in selected cases only (e.g., when the information needs to reach destinations such as remote servers, for permanently storing the gathered information).

Since the service is the original goal of the network, we let the service itself define how the network is supposed to be in order to satisfy its requirements. Networking will occur only as a consequence of service needs and the network itself will evolve and adapt together with the service. In the envisioned ubiquitous scenario the environment will dictate the principal rules of adaption. Users will be mobile and will change their location in short periods of time, leading to a continuously changing environment. The success of the service will be in following these changes and in subsequently adapting its main functionalities. In this sense the network may be interpreted as the habitat where organisms are moving and the genetic information codes their behavior and goals.

\section{BIONETS}

\subsection{Related Work}

Several examples are available in the literature, where biological concepts are considered as models to imitate. Each one of this examples focuses on a different biological aspect and apply it to solve or to optimize a specific technological problem.

In [6] 7] the swarm intelligence of social insects has inspired an evolutionary framework capable of connecting heterogeneous objects and services. This dis- 
tributed framework should exploit the decentralized organization of autonomous biological individuals leading to an emergent behavior. According to these principles in 8 , the described framework has been implemented and evaluated in terms of messages delivery efficiency and high workload situations.

In 9] autonomic techniques are applied to system management, where a centralized management approach is unlikely to be adopted due to the enormous number of devices participating in large-scale pervasive scenarios. Drawing inspiration from nature, problems with real-world relevance are approached. For example, in Flyphones the autonomic creation of cells macro-structure in animals cells is used to determine the channel allocation algorithm in a mobile telephone network, while a bacterium- inspired software has been developed for the adaptive management of active service networks.

In the above mentioned examples the genetic approach is not considered. Since in nature the genes are the coding blocks of an unbounded set of selforganizing behaviors, this is what we would like to reproduce through the BIONETS approach. Different coded behaviors can then be naturally chosen by the natural selection.

\subsection{BIONETS Principles}

In the depicted scenario services are associated with living organisms. Service is defined by chromosomes. In this way service evolves and adapts to the environment constantly and autonomously, Chromosomes are collections of genes that are the smallest service (related) data unit and inborn intelligence/instincts and thus represent all the information needed for the organism to function and service to be executed.

As in nature, it is possible to define a complete life-cycle of the organisms and therefore of services. The life cycle starts from the birth of an organism, goes through the reproduction and ends with the death. Each one of this stages will be defined in the following. Reproduction and evolution occur applying evolution rules inherited from nature.

Fitness is measuring the correspondence of the organism genetic information with the environment and determines the exchange of information(genetic information). Therefore no end to end communication concept exists in these systems, information is only exchanged as needed, locally, between mating organisms. Environment is determining the Natural Selection based on the fitness of the organisms with the environment leading to the best services possible as a function of the environment.

The Service Is the Organism. We are envisioning a scenario where users will be more and more interested in a service able to provide reliable localized information. The role of the service will be for instance to provide answers to questions like How is the weather around the train station? or Where will I find a free parking space around there?. Services will be hosted on users' devices and will go around through the physical movement of the users. 
Each service is constituted by a program and its related data that is organized into chromosomes.

The Chromosome. The service will use his genetic information to run its main functionalities. The genetic information is stored in the chromosome. Each chromosome consists of:

- Data that is the genetic information of the organism, organized in genes

- A plugin that stores a syntax notation describing the actions dictated by the chromosome and the fitness (degree of attraction) operator yielding natural selection through preferred mating

The Gene. Genes are a tuple of information and consist of:

- value

- timing information

- information source ID / location information

- other data depending on the service

Organisms are diploid, meaning that there will always be 2 homologous chromosomes associated to each service. The two homologous chromosomes will have the same genes but in different forms. Alleles are different forms of the same gene and corresponds to different values in the tuple of gene information. They may differ in timing information or in the source value information. Each allele may be dominant or recessive depending on the service behavior.

Having two chromosomes allows us to estimate the reliability of the data. We would probably always choose the youngest data value to be the actual one if it is a parking lot, but we might also average the sensor data if it represents temperature. The choice of the preferred value among the two reflects the concept of dominant and recessive genes.

As in nature, recessive information will enable the service to survive in different environments, providing the service with higher resilience against data corruption and fraud and may even allow for additional features.

\subsection{Service Life Cycle}

A service is born when the user downloads the chromosome onto his device. From that moment on, the user is able to interact with the other organisms (i.e. users carrying chromosomes) and with the environment where the users are physically moving. When gathering information from the environment the service grows. While growing, the service improves its functionalities, meaning that it becomes able to increase performance.

When a user meets another user while moving, services may reproduce and produce offsprings. It is in this phase that evolution and natural selection occur. In order to be able to reproduce the service must satisfy some fitness require- 
ments, since it is not willing to spread useless information. We assume a service to be dead when he can not reproduce anymore.

Birth. The service is born when the user gets (downloads) an empty chromosome of a certain service that consists only of the plugin. From now on the user can read data from sensors and use the syntax definition from the plugin. Note that the user is haploid now, i.e. it has only one chromosome per service.

Growth. When reading sensor data, the user fills the chromosomes both at the same time. This information is in any case more reliable than the previous information on the chromosomes.

Reproduction. The concept of mating is performed the following way: In Meiosis, the diploid cell splits into 2 haploid reproductive cells (the eggs or sperms) 1 . This means that the chromosome pair is being split and copied. Two packets are sent out one after another containing one chromosome each. In the best case when all the sent packets reach the receiver, it has 4 different combinations of the chromosome pairs. Note that this is the best case, the user may for energy reasons decide to send out only one chromosome, or receive only one because of packet loss.

A selection has to take place to decide which of these combination survives. This selection could be influenced by the quality of the plugin (by the version number). Having such a selection can help to spread new versions of plugins. It also may help to repair broken chromosomes (that were damaged during the wireless transmission). In a sense, we allow for truly spontaneous mutations and we may even find that mutations spread. It remains to be seen if this is any good.

The selection occurs as a consequence of localization and age. We can define the fitness of a chromosome as the average of the timing information of the genes weighted with the localization information. In this sense the environment participates in the selection, since the survival of a service also depends from where the user is (but not only) when he mate with another user.

Death. If the sensor data in the chromosome is too old, it is very likely useless. Thus we forbid to send out the chromosome after a certain threshold (coded in the plugin). This way the chromosome can die, but it may be reborn through reading new sensor data or receiving a fresh chromosome.

The service is considered alive as long as it is able to answer to questions. Death is therefore a consequence of outdated chromosomal information (sensor gathered information is aging). It is in the interest of the user to exchange information and to gather sensor information and this same interest drives the service instinct to survive. We have now defined a complete life cycle of the service.

\footnotetext{
${ }^{1}$ In real life 4 haploid cells would appear because of one step of copying the chromosomes including cross-over. Each two copies would be identical.
} 


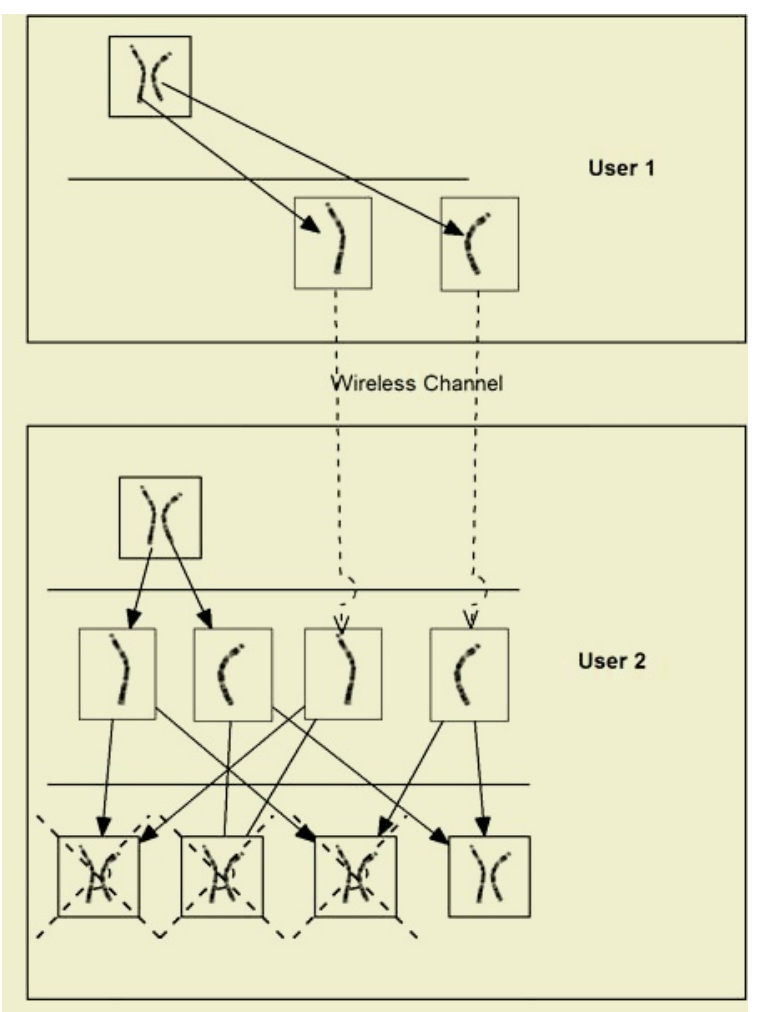

Fig. 1. Mating process

\section{Evolution of the Service}

The basic principles described in chapter 3 together with the rules of the selection determine the evolution of the service. In the model we have identified the issue of the instinct to survive, which is however is still an open question.

Since the service's aim is to provide a measurable benefit to the user, this benefit should be the metric to determine the fitness of a certain service. Thus, the simplest fitness criterion is: "User likes the service". This opinion could be measured by explicit user feedback, but other ways of inferring it from the user's behavior should be found.

We are expecting the service to carry some memory on what has determined his successfulness. Hence, the chromosomes will contain not only raw data, changing continuously but also some coded instructions or intelligence.

\section{Conclusions and Future Work}

We have presented a new concept of information exchange in pervasive networks. The major conceptual shift is the use of networks of occasional information ex- 
changes between mobile users, helping to spread information rather than forwarding data packets. The use of genetic models leads to a population of service instances on a set of user nodes. This population can grow if the service is successful or decline if it is not. In addition to the growth of populations BIONETS allow for the evolution of the service itself through mutations and selection of the fittest. The specific fitness criteria are still to be elaborated.

In order to examine the behavior of the proposed model in a day-to-day scenario we consider the case of a parking lot application. In such scenario the city is split into blocks and each gene will contain the information on the status of a parking spot (FREE or OCCUPIED). The service will guide the users towards the nearest free parking place and will evolve according to the environment where the users are moving. Since first simulations indicated the great potential of this idea, we are expanding the model towards more realistic user behavior right now.

\section{References}

1. Chlamtac, I., Carreras, I., Woesner, H. In: From Internets to BIONETS: Biological Kinetic Service Oriented Networks. Springer Science (2005) 75-95

2. R.C. Shah, S. Roy, S.J., Brunette, W.: Data mules: modeling a three-tier architecture for sparse sensor networks. In: Proceedings of the IEEE Workshop on Sensor Network Protocols and Applications (SNPA). (2003) 30-41

3. Zhao, W., Ammar, M., Zegura, E.: A message ferrying approach for data delivery in sparse mobile ad hoc networks. In: Proceedings of the 5th ACM international symposium on Mobile ad hoc networking and computing, ACM Press (2004) 187198

4. Vahdat, A., Becker, D.: Epidemic routing for partially connected ad hoc networks. Technical Report CS-200006, Duke University (2000)

5. Grossglauser, M., Tse, D.N.C.: Mobility increases the capacity of ad hoc wireless networks. IEEE/ACM Trans. Netw. 10 (2002) 477-486

6. Nakano, T., Suda, T.: Adaptive and evolvable network services. In Kalyanmoy Deb, e.a., ed.: Genetic and Evolutionary Computation GECCO 2004. Volume 3102/2004., Springer-Verlag Heidelberg (2004) 151-162

7. Wang, M., Suda, T.: The bio-networking architecture: A biologically inspired approach to the design of scalable, adaptive, and survivable/available network applications. In: SAINT. (2001) 43-

8. Suzuki, J., Suda, T.: Design and implementation of an scalable infrastructure for autonomous adaptive agents. In: Proc. of the 15th IASTED International Conference on Parallel and Distributed Computing and Systems. (2003)

9. Shackleton, M., Saffre, F., Tateson, R., Bonsma, E., Roadknight, C.: Autonomic computing for pervasive ict a whole-system perspective. BT Technology Journal 3 (2004) 